\title{
One-Step Electrodeposited 3D-Ternary Composite of Zirconia nanoparticles, rGO and PolyPyrrole with Enhanced Supercapacitor Performance
}

Ana Paula P. Alves ${ }^{1,2}$, Ryota Koizumi ${ }^{1}$, Atanu Samanta ${ }^{3}$, Leonardo D. Machado ${ }^{4,5}$, Abhisek Singh $^{3}$, Douglas S. Galvao ${ }^{4}$, Glaura G. Silva ${ }^{2}$, Chandra S. Tiwary ${ }^{1}$, and Pulickel M. Ajayan $^{1 *}$

${ }^{1}$ Department of Materials Science and NanoEngineering, Rice University, Houston, Texas, USA-77005.

${ }^{2}$ Departamento de Química, Universidade Federal de Minas Gerais. Av. Antônio Carlos, 6627, Pampulha, 31270-901, Belo Horizonte - MG, Brazil.

${ }^{3}$ Materials Research Centre, Indian Institute of Science, Bangalore, India-560012.

${ }^{4}$ Departamento de Física Aplicada, Universidade Estadual de Campinas, Campinas-SP, 13083-959, Brazil.

${ }^{5}$ Departamento de Física Teórica e Experimental, Universidade Federal do Rio Grande do Norte, Natal-RN, 59072-970, Brazil

\begin{abstract}
Supercapacitor electrodes consisting of conjugated polymers (CP), metal oxides and graphene nanosheets have been explored as a strategy to achieve high specific capacitance, power, energy density, and stability. In this work, we synthesized a 3D structure composed of zirconia oxide nanoparticles $\left(\mathrm{ZrO}_{2}\right)$, reduced graphene oxide ( $\left.\mathrm{rGO}\right)$ and polypyrrole (PPy), using a simple and easily scalable one-step chronoamperometry method. Detailed characterization revealed that the addition of $\mathrm{rGO}$ and $\mathrm{ZrO}_{2}$ modified the morphology of the electrode material. The capacitance of the resulting architecture improved by up to a $100 \%$. The ternary composite featured high stability, with an increase of 5\% in capacitance after a thousand cycles. DFT and MD simulations were carried out in order to provide further insight on the role of zirconia.
\end{abstract}

Keywords: Electrodeposited ternary composite; $\mathrm{ZrO}_{2} / \mathrm{rGO} / \mathrm{PPy} ; 3 \mathrm{D}$-ternary electrode; Supercapacitor; DFT; MD simulation.

* Corresponding authors

E-mail: glaura.goulart@gmail.com, cst.iisc@gmail.com; ajayan@rice.edu

Phone: +1 713-348-5904/ Fax: +1 713-348-5423 


\section{Introduction}

Supercapacitors are energy storage devices capable of releasing large amounts of energy in short time periods, providing high power and energy densities for applications demanding rapid and intense electrical pulses [1]. Carbon nanomaterials based supercapacitors offer advantages in terms of capacitance, energy, and power density, due to their high surface area, porosity, and electrical conductivity [2, 3]. In particular, graphene-based supercapacitors enable device miniaturization combining high performance with thermal stability and flexibility $[4,5]$.

At the same time, in these devices the energy storage mechanism is purely electrostatic, generated by the formation of an electrical double layer (EDL) on the electrodes' surface. It can then be advantageous to incorporate materials that undergo reversible oxidation/reduction reactions, in order to further increase capacitance values. Typical materials include conjugated polymers or metal oxides, but they usually have low electrochemical stability [6, 7]. To address this issue, a strategy is to engineer ternary composites, combining graphene with two different kinds of materials [8-15]. The resulting 3D nano-architectures present better cycling stability along with high specific capacitance, and other improved electrochemical properties $[8,10]$. Several groups have studied ternary composites for use in supercapacitors, like graphene- $\mathrm{SnO}_{2}$-polyaniline [8], $\mathrm{MnFe}_{2} \mathrm{O}_{4}$-graphene-polyaniline [10], $\mathrm{MnO}_{2}$ nanorodsgrapheneoxide-polyaniline [11], graphene- $\mathrm{SnO}_{2}-\mathrm{PPy}$ [13]. Ternary composites based on oxides of Zirconium, however, have yet to be more explored in supercapacitors [16].

Zirconia nanoparticles have high thermal stability, and can be used as a reinforcement material to improve mechanical and thermal properties [17-19]. Moreover, zirconia 
nanoparticles have been shown to interact strongly with rGO sheets, and blends of these two materials can be used to create 3D interconnected composites, with high porosity [20]. Present electrochemical applications for zirconia and graphene (or GO) composites include sensors and capacitor [21-23].

In this work, we used the chronoamperometry method to perform a one-step electrochemical deposition, that yielded a highly porous $3 \mathrm{D}$ structure, consisting of $\mathrm{ZrO}_{2}$ nanoparticles, reduced graphene oxide, and in-situ polymerized pyrrole (PPy). This ternary architecture, grown on a carbon fiber paper, features high conductivity and surface area, and was used as a current collector electrode with high specific capacitance, without the inclusion of binders or conductive additives. We investigated varied combinations and ratios of individual components, and employed detailed spectroscopy, microscopy, and simulations to understand the results. We also performed cyclic tests to evaluate material stability, and then examined the changes in material morphology.

\section{Experimental Section}

\subsection{Synthesis of ternary and binary composites and pure PPy electrodes}

Graphene oxide (GO) was synthesized from graphite (Bay Carbon, Inc.) by a modified Hummers method, as described in the literature [24]. The 3D ternary composite $\mathrm{PPy} / \mathrm{rGO} / \mathrm{ZrO}_{2}$ was synthesized by the electrodeposition (chronoamperometry) method, which consists of electrochemical polymerization of the pyrrole monomer, electrochemical reduction of GO, and the formation of $\mathrm{ZrO}_{2}$ nanoparticles. The current collector was carbon fiber paper (Spectracarb 2050A-1050, Engineered Fibers Technology- Fuell Cell Store) with electrical 
resistivity (through plane) of $18 \mathrm{~m} \Omega \mathrm{cm}$ and (in-plane) of $5.4 \mathrm{~m} \Omega \mathrm{cm}$. The reference electrode was $\mathrm{Ag} / \mathrm{AgCl}$ and the counter electrode was a platinum wire. $1 \mathrm{mg} \mathrm{mL}^{-1}$ of $\mathrm{GO}$ in aqueous dispersion was mixed with $0.1 \mathrm{~mol} \mathrm{~L}^{-1}$ of pyrrole (Sigma Aldrich, reagent grade $98 \%$ ), previously purified by distillation, $7 \mathrm{mmol} \mathrm{L}^{-1}$ of zirconyl chloride octahydrate (Sigma Aldrich, reagent grade $98 \%$ ), and $0.1 \mathrm{~mol} \mathrm{~L}^{-1}$ of sodium para-toluene sulfonate (NaPTS, Sigma Aldrich), in $50 \mathrm{~mL}$ of deionized water. We then sonicated the solution for an hour at room temperature. The electrodeposition of the electrodes was performed for 30 minutes by application of $+0.8 \mathrm{~V}$ and then $-1.1 \mathrm{~V}$ during 60 seconds for electrodeposition of $\mathrm{ZrO}_{2}$ [25]. The electrodes have approximately $3 \mathrm{mg}$ of total weight and $30 \mu \mathrm{m}$ of thickness. For comparative purposes, the pure PPy and the PPy/rGO were also prepared by applying $+0.8 \mathrm{~V}$ during 30 minutes at the same concentration of the solutions. After electrodeposition, the prepared electrodes were vigorously washed with deionized water and were heated in a vacuum oven at $60{ }^{\circ} \mathrm{C}$ for 12 hours.

\subsection{Material characterization and supercapacitor measurements}

The ternary and binary composites, as well as the polypyrrole, were investigated by Xray photoelectron spectroscopy (XPS, PHI Quantera XPS) on a PHI-5000C ESCA system with Al KR X-ray as an excitation source. The morphology of the samples was observed on the surface of deposits using FEI Quanta 400 scanning electron microscope under high vacuum at $10 \mathrm{kV}$. Raman spectra were taken in a Renishaw spectrometer with a 633-nm laser and a 20 x objective. TEM images were taken using a JEOL 2100 field emission gun transmission electron microscope. For preparing the TEM samples, the powder samples were 
bath sonicated in isopropyl alcohol for $30 \mathrm{~min}$. A few drops were then cast onto the holycarbon grid and allowed to dry in vacuum overnight.

Electrochemical experiments were carried out in a typical three-electrode cell using Autolab Potentiostat Galvanostat (Autolab PGSTAT 302N). The tests were conducted in 1 mol $\mathrm{L}^{-1} \mathrm{KCl}$ electrolyte with the fabricated electrode as working electrode, $\mathrm{Ag} / \mathrm{AgCl}$ as reference and $\mathrm{Pt}$ wire as counter electrode. The charge/discharge electrochemical tests were performed at current densities of $0.75,1$ and $2 \mathrm{~A} \mathrm{~g}^{-1}$ in the voltage range from -0.5 to $0.5 \mathrm{~V}$. The cyclic voltammetric tests were carried out at scan rate of $80,50,30,10$ and $5 \mathrm{mV} \mathrm{s}^{-1}$. The tenth cyclic voltammetric and charge/discharge curves were collected for each scan rate and the last cycle was used for the capacitance calculations. The stability of the capacitors was evaluated over 1000 cycles at room temperature and $1 \mathrm{~A} \mathrm{~g} \mathrm{~g}^{-1}$ current density in a twoelectrodes system, where two-electrodes with the same mass are isolated by a separator (Whatman QM-A Quartz Microfiber Filters 300-350 $\mu \mathrm{m}$ ). The impedance spectra were recorded at $0 \mathrm{~V}$, by applying a signal of $5.0 \mathrm{mV}$ of amplitude over the frequency range from 1 $\mathrm{MHz}$ to $0.01 \mathrm{~Hz}$ vs OCP.

\subsection{Methodology of ab-initio theoretical study}

The ground-state calculations were carried out using first-principles spin polarized density functional theory (DFT) as implemented in the Vienna Ab Initio Simulation Package (VASP) [26, 27]. The ionic cores were described by all-electron projector augmented wave potentials, and the electronic exchange and correlation was described using the PerdewBurke-Ernzerhof (PBE) generalized gradient approximation (GGA) [28, 29]. To incorporate long-range vdW interactions, Grimme's DFT-D2 (where D2 refers to the second generation 
of this method) approach is used for all calculations, as implemented in VASP [30, 31]. In order to simulate $\left(\mathrm{K}, \mathrm{Cl}, \mathrm{ZrO}_{2}\right.$ nanoparticle)/graphene system, a supercell of 9x9x1 graphene sheet is used, which is big enough to make sure that the distance between $\mathrm{ZrO}_{2}$ nanoparticle is at least $15 \AA$. A vacuum of $30 \AA$ is used along the out of plane direction to avoid the interaction among the images. In the calculations was used a plane-wave cutoff of $400 \mathrm{eV}$. The structures were optimized by minimizing the forces on each atom to below $0.05 \mathrm{eV} \AA^{-1}$. The Brillouin zone was sampled with $3 \times 3 \times 1$ Monkhorst- Pack k-mesh for all the selfconsistent calculations [32].

\subsection{Methodology of Molecular Dynamics simulations}

The annealing-like process consisted of six steps, and along each of these steps a chain of three Nosé-Hoover thermostats and barostats was employed. Target pressure for the barostat was always 0 atm. In order, the steps were: (1) Minimize a periodic GO sheet for 2000 steps. (2) Equilibrate the sheet for 50000 steps at 300 K. (3) Set a heating ramp lasting 400000 steps with a final temperature of 1300 K. (4) Equilibrate the system for 200000 steps at $1300 \mathrm{~K}$. (5) Set a cooling ramp lasting 300000 steps with a final temperature of $300 \mathrm{~K}$. (6) Equilibrate the system for 50000 steps at $300 \mathrm{~K}$.

Although the annealed sheet was periodic, during our compression and torsion tests we removed periodicity, and employed non-periodic boundary conditions. During the thermalization step preceding these tests, we also used springs to prevent atoms in the green region from moving up/down or left/right. This was important in the torsional test to prevent the rotation axis from becoming askew. 


\section{Results and discussion}

\subsection{Characterization of the materials}

A schematic view of the process used to produce the electrodes is presented in Figure 1a, along with representative samples of the unary (PPy), binary (PPy/rGO), and ternary $\left(\mathrm{PPy} / \mathrm{rGO} / \mathrm{ZrO}_{2}\right)$ electrodes. A detailed description of the synthesis process can be found in the experimental section. Figure $1 \mathrm{~b}$ shows the Raman spectra for PPy, PPy/rGO and $\mathrm{PPy} / \mathrm{rGO} / \mathrm{ZrO}_{2}$. The PPy spectra exhibits characteristic peaks at $1583 \mathrm{~cm}^{-1}(\mathrm{C}=\mathrm{C}$ backbone stretching mode), 1384 and $1311 \mathrm{~cm}^{-1}$ (C-N ring stretching mode and $\mathrm{N}-\mathrm{H}$ in-plane deformation of the doped species), 1079 and $1052 \mathrm{~cm}^{-1}$ (C-H in-plane deformation mode). The Raman modes observed in 973 and $934 \mathrm{~cm}^{-1}$ are due to polaron and bipolaron symmetric $\mathrm{C}-\mathrm{H}$ in plane bending, respectively $[33,34]$. The incorporation of rGO into the system results in an increased contribution of the D and G vibrational modes, hampering the visualization of the polymer peaks. The same behavior was observed in the spectra of the ternary composite.

We also carried out X-ray photoelectron spectroscopy (XPS) characterization to confirm the composition of the fabricated electrodes (Figure 1c). All of the samples showed C $1 \mathrm{~s}, \mathrm{O} 1 \mathrm{~s}$ and $\mathrm{N} 1 \mathrm{~s}$ photoemission peaks at $\sim 284.4 \mathrm{eV}, \sim 532.1 \mathrm{eV}$ and $\sim 398.8 \mathrm{eV}$, respectively. We also observed the $S 2 p$ peak in all samples, possibly because the ionic species of the supporting electrolyte doped the polymer during synthesis. $\mathrm{Zr}$ peaks were observed as $3 \mathrm{~d}$-core level at $182.3 \mathrm{eV}\left(3 \mathrm{~d}_{5 / 2}\right)$ and $184.7 \mathrm{eV}\left(3 \mathrm{~d}_{3 / 2}\right)$, which is expected according to the energy splitting of the standard spectrum of $\mathrm{ZrO}_{2}$ nanoparticles [35]. Other $\mathrm{Zr}$ peaks were observed, at $\sim 332 \mathrm{eV}\left(3 \mathrm{p}_{1 / 2}\right.$ and $\left.3 \mathrm{p}_{3 / 2}\right), 433 \mathrm{eV}(3 \mathrm{~s})$, and $29 \mathrm{eV}(4 \mathrm{p})$. The element content as determined 
by the high-resolution XPS analysis for both the PPy and the composites is presented in Table 1.

The photoelectron spectra of ternary composite were de-convoluted into Gaussian and Lorentzian peaks. The $\mathrm{C} 1 \mathrm{~s}$ photoelectron peaks (Figure 1d) can be associated to C-C species at $284.4 \mathrm{eV}, \mathrm{C}-\mathrm{O}$ (related to non-reduced species in rGO or polymer oxidized moieties) and $\mathrm{C}-\mathrm{N}$ (PPy rings) at $285.6 \mathrm{eV}, \mathrm{C}=\mathrm{N}$ and polaron $=\mathrm{C}-\mathrm{NH}^{\cdot+}$ (PPy rings), hydroxylated defects $\mathrm{C}-\mathrm{OH}$ in $\mathrm{rGO}$ and $\mathrm{C}-\mathrm{S}$ (anion dopant) at $286.6 \mathrm{eV}$ and bipolarons $-\mathrm{C}=\mathrm{N}^{+}$(PPy) and carbonylic defects (rGO) at $288.3 \mathrm{eV}[13,36]$. The $\mathrm{N}$ 1s peak showed three XPS lines: at $399.8 \mathrm{eV}$ (neutral N-H in PPy rings), $400.5 \mathrm{eV}$ (the positively species or polaron) and 401.7 eV (bipolaron species) [12, 36].

The $\mathrm{O} 1 \mathrm{~s}$ peaks displayed two contributions in pure PPy at 531.6 and $533.0 \mathrm{eV}$. The first one can be attributed to $\mathrm{O}=\mathrm{S}$ from anion dopant and the second one to chemisorbed oxygen and polymer oxidized moieties $[36,37]$. These peaks were also observed in the binary composite at 531.1 and $535.1 \mathrm{eV}$, and in the ternary composite at 531.2 and $535.7 \mathrm{eV}$. One photoelectron peak at $\sim 532.4 \mathrm{eV}$ was found in both composites that represents $\mathrm{C}=\mathrm{O}$ and $\mathrm{O}-\mathrm{H}$ species [37]. Besides that, two contributions appeared in $\mathrm{PPy} / \mathrm{rGO} / \mathrm{ZrO}_{2}$ spectra: $530.3 \mathrm{eV}$ corresponding to the $\mathrm{Zr}-\mathrm{O}$ bond and $534.0 \mathrm{eV}$ corresponding to $\mathrm{C}-\mathrm{O}$ bonds [38]. The photoelectron spectra of the binary composite and PPy were deconvoluted, and are shown in Figure S1 (Supporting Information).

The electrodes were observed at different magnifications using Scanning Electron Microscope (SEM), as shown in Figure 2a-d. Pure PPy presents itself as a smooth coating on the carbon fiber substrate (Figure 2a) with the presence of cracks (shown in the high 
magnification image in the inset). For the rGO reinforced PPy film, the morphological structure was modified to form a granular and porous three-dimensional structure (Figure $2 b$ ). The granules of rGO are uniformly distributed on the film and no crack or separation is observed in the composite film. In the ternary composite images (Figure 2c-d), the zirconia nanoparticles on the PPy/rGO can be observed. This morphology shows a foliate feature, which is different from the granular aspect of the binary composite. Even in small content, the addition of $\mathrm{ZrO}_{2}$ particles lead to changes in the structural morphology and porosity of the electrodes. Bright field TEM (transmission electron microscope) images of $\mathrm{rGO} / \mathrm{ZrO}_{2}$ can be visualized in Figure 2e, and show zirconia nanoparticles distributed on the rGO sheets. The SAED (selected area electron diffraction) pattern covering sheet and nanoparticle confirms the presence of $\mathrm{ZrO}_{2}$ (shown as inset of Figure 2e). The morphology of rGO sheets reinforced with $\mathrm{ZrO}_{2}$ nanoparticle is similar to that reported in literature [20]. The HRTEM (high resolution TEM) image of the particle, Figure $2 \mathrm{f}$, confirms the nanoparticle is monoclinic. The Fast Fourier Transform (FFT) of the lattice image shows 110 monoclinic $\mathrm{ZrO}_{2}$ nanoparticles, and the atomic arrangement is shown as inset.

\subsection{Electrochemical measurements}

The performance of unary (PPy), binary (PPy/rGO), and ternary $\left(\mathrm{PPy} / \mathrm{rGO} / \mathrm{ZrO}_{2}\right)$ electrodes were evaluated and compared. The CV profile indicates the mechanism of energy storage is that of a capacitor [3]. In Figure 3a, CV curves show a capacitive behavior for PPy, due to the faradaic contribution of the polymer redox reactions [6]. For the binary composite, the addition of rGO leads to a larger box-like area, attributable to double layer contributions. The maximum capacitance value was obtained with a $5 \mathrm{mV} \mathrm{s}^{-1}$ scan rate: $213.7 \mathrm{~F} \mathrm{~g}^{-1}$ for 
PPy/rGO and $171.1 \mathrm{~F} \mathrm{~g}^{-1}$ for PPy. With the incorporation of $\mathrm{ZrO}_{2}$ nanoparticles in the system, the box-like area of the curve increased even more, reaching $341.0 \mathrm{~F} \mathrm{~g}^{-1}$ at $5 \mathrm{mV} \mathrm{s}^{-1}$, which is 1.6/2 times higher than the binary/unary system. The $\mathrm{ZrO}_{2}$ nanoparticles also help to inhibit the agglomeration of rGO nanosheets and consequently, to improve the electrolyte access to the active sites of the electrodes [16, 23]. Furthermore, the effective change observed in SEM images for the ternary composite shows that the porosity and the morphological structure have been improved by the presence of the $\mathrm{ZrO}_{2}$. The material with high specific surface area and high porosity at the nanoscale level changes the electrochemical behavior facilitating the transport of ions through the active material [16]. The effect of $\mathrm{ZrO}_{2}$ on the morphology and structural integrity is also another important aspect which is discussed in subsequent sections.

The comparative results are shown in Figure 3b. Figure S2 (Supporting Information) shows CV curves of ternary composites at different scan rates. We observed the box-like profile until $30 \mathrm{mV} \mathrm{s}^{-1}$, and a pseudocapacitive profile became apparent at the higher scan rates. At these rates, not all of active material can react and interact with the electrolyte ions [39].

The galvanostatic charge/discharge curves are presented in Figure $3 \mathrm{c}$ for PPy, PPy/rGO, and $\mathrm{PPy} / \mathrm{rGO} / \mathrm{ZrO}_{2}$ at a current density of $1 \mathrm{Ag}^{-1}$. The curves profile show a triangular shape, indicative of a capacitive behavior and good coulombic efficiency. Note that the voltage (IR drop) is lower in the ternary composite, which is in agreement with the results obtained by EIS and CV methods [39]. Results for the ternary composite at different current densities are presented in Figure S2 (Supporting Information). Specific capacitances values could be calculated by $C_{s p}=\frac{I}{m \frac{d V}{d t}}$, where, I is the applied current and $d V / d t$ is the slope of the 
discharge curve calculated in the upper half of the curve after the IR drop, where $^{d V} / d t=\left(V_{\max }-1 / 2 V_{\max }\right) /\left(t_{2}-t_{1}\right)[40]$.

Specific capacitance increased from $143.1 \mathrm{~F} \mathrm{~g}^{-1}$ for pure PPy, to $200.1 \mathrm{~F} \mathrm{~g}^{-1}$ for $\mathrm{PPy} / \mathrm{rGO}$, and then to $279.2 \mathrm{~F} \mathrm{~g}^{-1}$ for $\mathrm{PPy} / \mathrm{rGO} / \mathrm{ZrO}_{2}$ electrodes. These specific capacitance results, presented in Figure 3d, are consistent with those obtained by the cyclic voltammetry method. Such large increases in capacitance can be attributed to the highly porous 3D structure that forms in the ternary case, where $\mathrm{ZrO}_{2}$ nanoparticles and connected in a network, which is embedded in a PPy matrix.

In order to collect Nyquist plots we used a three-electrode system, with an aqueous 1 mol L ${ }^{-1} \mathrm{KCl}$ solution as electrolyte - see Figure 3e. The curves' profile showed a semicircle with low resistance for all materials, followed by a linear region at low frequencies with an angle of incline close to $90^{\circ}$, indicative of capacitor behavior [40]. In an intermediary frequency region, we observed a line with an angle of incline of approximately $45^{\circ}$, which can be correlated to diffusion processes occurring in the porous structure of these systems. The point at intermediate frequency where the semicircle in the plot intercepts the x-axis can be used to determine the equivalent series resistance (ESR), representing the electrode material-electrolyte interface resistance. We obtained very low values for all systems: $3.7 \Omega$ for both the unary and binary systems, and $2.2 \Omega$ for the ternary case. From this result, we conclude that zirconia lowers the change transfer resistance. We could not observe the point in which the plot intercepts the x-axis at high frequencies, which provides the electrolyte resistance. The point at intermediate frequency where the semicircle in the Nyquist plot intercepts the $\mathrm{x}$-axis can be used to determine charge transfer resistance $\left(\mathrm{R}_{\mathrm{CT}}\right)$. We obtained 
very low values for all systems: $3.7 \Omega$ for both the unary and binary systems, and $2.2 \Omega$ for the ternary case. From this result, we concluded that zirconia lowers the charge transfer resistance. We could not observe the point in which the semicircle intercepts the $\mathrm{x}$-axis at high frequencies, which provides the electrolyte resistance and the contact resistance. The high frequency data were probably disturbed by instrumental artifacts, such as parasitic capacitance and inductance or reference electrode limitations [41].

To verify cycling stability, we employed the charge/discharge method at $1 \mathrm{~A} \mathrm{~g}^{-1}$ current density, in a two-electrodes system setup (Figure 3f). After 1000 cycles, both binary and ternary composites showed increase in specific capacitance values, which were $5 \%$ higher than initial values. The capacitance improvement could be related to a gradual wetting process of the electrodes, which enables its access to electrolyte ions through diffusion. This, in turn, leads to an IR drop (resistivity) decrease and to a consequent increase in capacitance [42].

This result corroborates the $\mathrm{CV}$ and $\mathrm{CD}$ measurements. The 3D architecture has a suitable morphology that allows a higher superficial area when compared to the binary composite, as observed in SEM images (Figure 2). The high electrical conductivity and porous structure provides easy access for the electrolyte to the electrodes surface, improving electrochemical properties. The ternary composite has a maximum energy density of $64 \mathrm{Wh}$ $\mathrm{kg}^{-1}$, at a power density of $2689 \mathrm{~W} \mathrm{~kg}^{-1}$.

It should be noted that other authors have obtained PPy ternary composites, like Salehifar et al. [39, 43]. These authors employed PPy/rGO/gold nanoparticles as electrodes and $0.1 \mathrm{~mol} \mathrm{~L}^{-1} \mathrm{H}_{2} \mathrm{SO}_{4}$ as electrolyte, and obtained capacitance values of $310 \mathrm{~F} \mathrm{~g}^{-1}$ by the $\mathrm{CV}$ method. For $\mathrm{Fe}_{2} \mathrm{O}_{3} / \mathrm{rGO} / \mathrm{PPy}$ electrodes, Eue et al. [25] obtained a specific capacitance value of $125.7 \mathrm{~F} \mathrm{~g}^{-1}$ and good stability (after 200 cycles, their material retained $81.3 \%$ of its initial 
capacitance). Other ternary systems have been studied, based on different polymers, such as polyaniline (PANI) and poly(3,4-ethylenedioxythiophene):poly(styrenesulfonate) (PEDOT:PSS) [10, 14, 44, 45]. Yan et al. [45] showed that $66.2 \%$ of the initial capacitance was retained after 2000 cycles for electrodes composed of $\mathrm{MnO}_{2} / \mathrm{rGO} / \mathrm{PEDOT}$ :PSS. Other authors also obtained ternary composites with good stability [14, 46, 47].

In order to evaluate the effect of cycling on the electrode structure, we obtained SEM images after these tests were completed. Results for the unary, binary, and ternary structures are shown, respectively, in Figure 4a-c. Lower and higher magnification images are presented, the latter as insets. Figure 4a reveals detachment and damage in some regions of PPy electrode, while imaging of the binary case (Figure $4 \mathrm{~b}$ ) reveals that the structure remained mostly intact. Results for the ternary case, presented in Figure 4c, display large absorption of electrolyte in the electrodes. To understand this result, observe the right insets at Figure 4a-c, in which we present contact angle measurements for the electrolyte on the electrodes. Contact angles under $90^{\circ}$ indicate the surface is wettable, and lower contact angles indicate better wettability. For the unary, binary, and ternary electrodes we obtained contact angles of $95^{\circ}$, $70^{\circ}$, and $57^{\circ}$. This result shows the electrolyte has better wettability in composite systems when compared to pure PPy. This result can also be correlated to the improved electrochemical performance of binary and ternary structures, as poor wettability can decrease the surface area available to the formation of the electric double layer [48].

The contact angle is related to the solid-liquid (electrode-electrolyte) interfacial energy $\left(\gamma_{\mathrm{SL}}\right)$ through Young's equation, and we employed density functional theory (DFT) to investigate how the addition of $\mathrm{ZrO}_{2}$ nanoparticles changes the interaction energy between the surface of the material and the electrolyte ions. Our calculations were carried out within the 
Vienna Ab Initio Simulation Package (VASP), and measured the interaction energy between isolated $\mathrm{K}$ and $\mathrm{Cl}$ atoms and zirconia nanoparticles/graphene [26, 27]. We have considered two cases: (1) 1 nm diameter $\mathrm{ZrO}_{2}$ particle supported by graphene, and (2) pristine graphene as shown in Figure 5a. The interaction energy at $0 \mathrm{~K}$ between atoms, particle and graphene substrate was calculated as follows

$$
E_{X}^{\text {int }}=E_{\text {substrate }+X}-\left(E_{\text {substrate }}+E_{X}\right) . \quad \text { Eq. } 1 .
$$

Where, $E_{\text {substrate }+X}$ is the total energy of the substrate with absorbed atom $(\mathrm{K}$ or $\mathrm{Cl})$ or $\mathrm{ZrO}_{2}$ particle, $E_{\text {substrate }}$ is the energy of the optimized bare substrate, and $E_{X}$ is the energy of the atom $(\mathrm{Cl}$ or $\mathrm{K})$ or the optimized particle in vacuum. Details on the DFT calculation are given in the theoretical method section. The calculated interaction energy is shown in Figure 5a. Interaction energy of $\mathrm{K}$ with pristine graphene is lower when compared to $\mathrm{Cl}$, indicating the former has stronger interaction with graphene. The interaction energy of $\mathrm{ZrO}_{2}$ nanoparticles with graphene is $-1.73 \mathrm{eV}$, which ensures they have good stability when supported on graphene. The interaction energy of $\mathrm{K}$ and $\mathrm{Cl}$ is significantly enhanced when a $\mathrm{ZrO}_{2}$ nanoparticle is added, as shown in Figure $5 \mathrm{~b} . \mathrm{K}$ favors the $\mathrm{O}$ site whereas $\mathrm{Cl}$ favors the $\mathrm{Zr}$ site of the $\mathrm{ZrO}_{2}$ nanoparticle, due to electronegative differences. The improved $\mathrm{ZrO}_{2}-\mathrm{K} / \mathrm{Cl}$ interaction should lower the solid-liquid (electrode-electrolyte) interfacial energy $\left(\gamma_{\mathrm{SL}}\right)$, and therefore the contact angle. This is in good agreement with experimental findings.

We also carried out Molecular Dynamics simulations to investigate whether the addition of zirconia nanoparticles modify the mechanical stability of the ternary composite. Incorporation of ions and solvating solution in the electrode material during cycling can lead 
to large changes in volume, which induce mechanical strain and stress [49]. The MD simulations were carried using the Reactive Force Field (ReaxFF) within the LAMMPS MD package [50-52]. A detailed account of these tests is presented in the Supporting Information. The setup used for compression/torsion tests is shown in Figure 5b/5d, and the results are presented in Figure 5c/5e. We observed that zirconia addition improved both compressive and torsional resistance for the composite material. The improved mechanical stability correlates well with the high stability of the ternary material during cycling.

\section{Conclusions}

In summary, this work reports a simple and easily scalable synthetic procedure to obtain an in-situ grown 3D-ternary composite consisting of polymerized PPy, electrochemically reduced $\mathrm{rGO}$ and nanoparticles of $\mathrm{ZrO}_{2}$. The incorporation of $\mathrm{rGO}$ and zirconia nanoparticles in the PPy matrix changed the morphological structure and improved the porosity of the active material, consequently increasing capacitance values by up to $100 \%$. The ternary supercapacitor device exhibited a capacitance of $341 \mathrm{Fg}^{-1}$ at a current density of $0.5 \mathrm{Ag}^{-1}$ and excellent cycle life stability: after a thousand cycles, the capacitance increased by $5 \%$. The DFT and MD simulations showed that $\mathrm{ZrO}_{2}$ addition improved composite/electrolyte interaction and mechanical stability of the architecture. This study can be further extended to design future energy storage materials engineered at atomistic level using bottom up approach with combination of different materials of metal, ceramic and polymers. 


\section{Acknowledgments}

Ana Paula P. Alves gratefully acknowledges the scholarship received from Conselho Nacional de Desenvolvimento Científico e Tecnológico (CNPq-Brazil). Glaura G. Silva thanks to FAPEMIG. A.K.S and A.S thanks Super Computer Education Research Centre, IISc, Bangalore, for providing the required computational facilities. LDM and DSG acknowledge Computational and financial support from the Center for Computational Engineering and Sciences at Unicamp through the FAPESP/CEPID Grant No. 2013/08293-7. LDM acknowledges financial support from the Brazilian Federal Agency CAPES via its PNPD program. C. S. Tiwary acknowledges US Department of Defense: the US Air Force of Scientific Research under the Project MURI: 'Synthesis and Characterization of 3D Carbon Nanotube Solid Networks’' Award No. FA9550-12-1-0035.

\section{References}

[1] J.R. Miller, P. Simon, Electrochemical Capacitors for Energy Management, Science 321 (2008) 651-652.

[2] E. Frackowiak, Carbon materials for supercapacitor application, Phys. Chem. Chem. Phys. 9 (2007) 1774-1785.

[3] B.E. Conway, Electrochemical supercapacitors: scientific fundamentals and technological applications, New York, 1999.

[4] L. Li, X. Zhang, J. Qiu, B.L. Weeks, S. Wang, Reduced graphene oxide-linked stacked polymer forests for high energy-density supercapacitor, Nano Energy 2 (2013) 628-635.

[5] A.S. Arico, P. Bruce, B. Scrosati, J.-M. Tarascon, W. van Schalkwijk, Nanostructured materials for advanced energy conversion and storage devices, Nat. Mater. 4 (2005) 366-377. [6] E. Frackowiak, V. Khomenko, K. Jurewicz, K. Lota, F. Béguin, Supercapacitors based on conducting polymers/nanotubes composites, J. Power Sources 153 (2006) 413-418. 
[7] J.Y. Hwang, M.F. El-Kady, Y. Wang, L. Wang, Y. Shao, K. Marsh, J.M. Ko, R.B. Kaner, Direct preparation and processing of graphene/ $\mathrm{RuO}_{2}$ nanocomposite electrodes for highperformance capacitive energy storage, Nano Energy 18 (2015) 57-70.

[8] Y. Jin, M. Jia, Design and synthesis of nanostructured graphene- $\mathrm{SnO}_{2}$-polyaniline ternary composite and their excellent supercapacitor performance, Colloids Surf. A Physicochem. Eng. Asp. 464 (2015) 17-25.

[9] L. Yu, M. Gan, L. Ma, H. Huang, H. Hu, Y. Li, Y. Tu, C. Ge, F. Yang, J. Yan, Facile synthesis of $\mathrm{MnO}_{2}$ /polyaniline nanorod arrays based on graphene and its electrochemical performance, Synth. Met. 198 (2014) 167-174.

[10] K.V. Sankar, R.K. Selvan, The ternary $\mathrm{MnFe}_{2} \mathrm{O}_{4} /$ graphene/polyaniline hybrid composite as negative electrode for supercapacitors, J. Power Sources 275 (2015) 399-407.

[11] G. Han, Y. Liu, L. Zhang, E. Kan, S. Zhang, J. Tang, W. Tang, $\mathrm{MnO}_{2}$ Nanorods Intercalating Graphene Oxide/Polyaniline Ternary Composites for Robust High-Performance Supercapacitors, Sci. Rep. 4 (2014) 4824-4830.

[12] X. Xia, Q. Hao, W. Lei, W. Wang, H. Wang, X. Wang, Reduced-graphene oxide/molybdenum oxide/polyaniline ternary composite for high energy density supercapacitors: Synthesis and properties, J. Mater. Chem. 22 (2012) 8314-8320.

[13] W. Wang, Q. Hao, W. Lei, X. Xia, X. Wang, Graphene/ $\mathrm{SnO}_{2} /$ polypyrrole ternary nanocomposites as supercapacitor electrode materials, RSC Adv. 2 (2012) 10268-10274.

[14] Y. Hou, Y. Cheng, T. Hobson, J. Liu, Design and Synthesis of Hierarchical $\mathrm{MnO}_{2}$ Nanospheres/Carbon Nanotubes/Conducting Polymer Ternary Composite for High Performance Electrochemical Electrodes, Nano Lett. 10 (2010) 2727-2733.

[15] P. Xiong, C. Hu, Y. Fan, W. Zhang, J. Zhu, X. Wang, Ternary manganese ferrite/graphene/polyaniline nanostructure with enhanced electrochemical capacitance performance, J. Power Sources 266 (2014) 384-392.

[16] S. Giri, D. Ghosh, C.K. Das, Growth of Vertically Aligned Tunable Polyaniline on Graphene/ZrO $\mathrm{Zr}_{2}$ Nanocomposites for Supercapacitor Energy-Storage Application, Adv. Func. Mater. 24 (2014) 1312-1324. 
[17] F. Cai, C. Jiang, Z. Zhang, E. Muttini, P. Fu, Y. Zhao, V. Ji, Fabrication and characterization of $\mathrm{Ni}-\mathrm{Zr}$ composite coatings using electrodepositing technique, J. Alloy Compd. 635 (2015) 73-81.

[18] Z. Chen, M. Zhang, W. Han, X. Wang, D. Tang, Electrodeposition of $\mathrm{Zr}$ and electrochemical formation of $\mathrm{Mg}-\mathrm{Zr}$ alloys from the eutectic $\mathrm{LiCl}-\mathrm{KCl}$, J. Alloy Compd. 459 (2008) 209-214.

[19] A. Nandy, C.S. Tiwary, A. Dutta, K. Chattopadhyay, S.K. Pradhan, Effect of Manganese (II) Oxide on microstructure and ionic transport properties of nanostructured cubic zirconia, Electrochim. Acta, 170 (2015) 360-368.

[20] D. Chakravarty, C.S. Tiwary, L.D. Machado, G. Brunetto, S. Vinod, R.M. Yadav, D.S. Galvao, S.V. Joshi, G. Sundararajan, P.M. Ajayan, Zirconia-Nanoparticle-Reinforced Morphology-Engineered Graphene-Based Foams, Adv. Mater. 27 (2015) 4534-4543.

[21] D. Du, J. Liu, X. Zhang, X. Cui, Y. Lin, One-step electrochemical deposition of a graphene-ZrO2 nanocomposite: Preparation, characterization and application for detection of organophosphorus agents, J. Mater. Chem. 21 (2011) 8032-8037.

[22] A.T. Ezhil Vilian, M. Rajkumar, S.-M. Chen, In situ electrochemical synthesis of highly loaded zirconium nanoparticles decorated reduced graphene oxide for the selective determination of dopamine and paracetamol in presence of ascorbic acid, Colloids Surf. B 115 (2014) 295-301.

[23] H. Mudila, S. Rana, M.G.H. Zaidi, Electrochemical performance of zirconia/graphene oxide nanocomposites cathode designed for high power density supercapacitor, J. Anal. Sci. Technol. 7 (2016) 3-14.

[24] D.C. Marcano, D.V. Kosynkin, J.M. Berlin, A. Sinitskii, Z. Sun, A. Slesarev, L.B. Alemany, W. Lu, J.M. Tour, Improved Synthesis of Graphene Oxide, ACS Nano, 4 (2010) 4806-4814.

[25] Y.C. Eeu, H.N. Lim, Y.S. Lim, S.A. Zakarya, N.M. Huang, Electrodeposition of Polypyrrole/Reduced Graphene Oxide/Iron Oxide Nanocomposite as Supercapacitor Electrode Material, J. Nanomater. 2013 (2013) 1-6.

[26] G. Kresse, J. Furthmüller, Efficient iterative schemes for ab initio total-energy calculations using a plane-wave basis set, Phys. Rev. B 54 (1996) 11169-11186. 
[27] G. Kresse, J. Furthmüller, Efficiency of ab-initio total energy calculations for metals and semiconductors using a plane-wave basis set, Comput. Mater. Sci. 6 (1996) 15-50.

[28] P.E. Blöchl, Projector augmented-wave method, Phys. Rev. B 50 (1994) 17953-17979.

[29] J.P. Perdew, K. Burke, M. Ernzerhof, Generalized Gradient Approximation Made Simple, Phys. Rev. Lett. 77 (1996) 3865-3868.

[30] S. Grimme, Semiempirical GGA-type density functional constructed with a long-range dispersion correction, J. Comput. Chem. 27 (2006) 1787-1799.

[31] T. Bučko, J. Hafner, S. Lebègue, J.G. Ángyán, Improved Description of the Structure of Molecular and Layered Crystals: Ab Initio DFT Calculations with van der Waals Corrections, J. Phys. Chem. A 114 (2010) 11814-11824.

[32] H.J. Monkhorst, J.D. Pack, Special points for Brillouin-zone integrations, Phys. Rev. B 13 (1976) 5188-5192.

[33] S.P. Lim, A. Pandikumar, Y.S. Lim, N.M. Huang, H.N. Lim, In-situ electrochemically deposited polypyrrole nanoparticles incorporated reduced graphene oxide as an efficient counter electrode for platinum-free dye-sensitized solar cells, Sci. Rep. 4 (2014) 5305-5312.

[34] G. Han, J. Yuan, G. Shi, F. Wei, Electrodeposition of polypyrrole/multiwalled carbon nanotube composite films, Thin Solid Films, 474 (2005) 64-69.

[35] S. Qu, S.-P. Pei, S.-L. Zhou, Y.-Y. Gu, One-Step Electrodeposited Carbon Nanotube/Zirconia/Myoglobin Film for Direct Electron Transfer and Electrocatalysis, J. Chin. Chem. Soc. 56 (2009) 822-827.

[36] N. Su, H. B. Li, S. J. Yuan, S. P. Yi, E. Q. Yin, Synthesis and characterization of polypyrrole doped with anionic spherical polyelectrolyte brushes, Express Polym. Lett. 6 (2012) 697-705.

[37] Y.J. Oh, J.J. Yoo, Y.I. Kim, J.K. Yoon, H.N. Yoon, J.-H. Kim, S.B. Park, Oxygen functional groups and electrochemical capacitive behavior of incompletely reduced graphene oxides as a thin-film electrode of supercapacitor, Electrochim. Acta 116 (2014) 118-128.

[38] J. Liu, M. Liao, M. Imura, A. Tanaka, H. Iwai, Y. Koide, Low on-resistance diamond field effect transistor with high-k $\mathrm{ZrO}_{2}$ as dielectric, Sci. Rep. 4 (2014) 6395-6400.

[39] N. Salehifar, J.S. Shayeh, S.O. Ranaei Siadat, K. Niknam, A. Ehsani, S. Kazemi Movahhed, Electrochemical study of supercapacitor performance of polypyrrole ternary 
nanocomposite electrode by fast Fourier transform continuous cyclic voltammetry, RSC Adv. 5 (2015) 96130-96137.

[40] M.D. Stoller, R.S. Ruoff, Best practice methods for determining an electrode material's performance for ultracapacitors, Energy Environ. Sci. 3 (2010) 1294-1301.

[41] F. Mansfeld, S. Lin, Y.C. Chen, H. Shih, Minimization of High-Frequency Phase Shifts in Impedance Measurements, J. Electrochem. Soc. 135 (1988) 906-907.

[42] A.A.-Z. Z. Jiang, S. Kawasaki, Unusual increase in the electric double-layer capacitance with charge-discharge cycles of nitrogen doped single-walled carbon nanotubes, Mater. Express 4 (2014) 331-336.

[43] J. Zhou, J. Lian, L. Hou, J. Zhang, H. Gou, M. Xia, Y. Zhao, T.A. Strobel, L. Tao, F. Gao, Ultrahigh volumetric capacitance and cyclic stability of fluorine and nitrogen co-doped carbon microspheres, Nat. Commun. 6 (2015) 8503-8511.

[44] R. Yuksel, H.E. Unalan, Textile supercapacitors-based on $\mathrm{MnO}_{2} / \mathrm{SWNT} /$ conducting polymer ternary composites, Int. J. Energ. Res. 39 (2015) 2042-2052.

[45] D. Yan, Y. Liu, Y. Li, R. Zhuo, Z. Wu, P. Ren, S. Li, J. Wang, P. Yan, Z. Geng, Synthesis and electrochemical properties of $\mathrm{MnO}_{2} / \mathrm{rGO} / \mathrm{PEDOT}$ :PSS ternary composite electrode material for supercapacitors, Mater. Lett. 127 (2014) 53-55.

[46] X. Lu, F. Zhang, H. Dou, C. Yuan, S. Yang, L. Hao, L. Shen, L. Zhang, X. Zhang, Preparation and electrochemical capacitance of hierarchical graphene/polypyrrole/carbon nanotube ternary composites, Electrochim. Acta 69 (2012) 160-166.

[47] S. P. Jose, C. S. Tiwary, S. Kosolwattana, P. Raghavan, L. D. Machado, C. Gautam, T. Prasankumar, J. Joyner, S. Ozden, D. S. Galvao, P. M. Ajayan, Enhanced supercapacitor performance of a 3D architecture tailored using atomically thin $\mathrm{rGO}-\mathrm{MoS}_{2} 2 \mathrm{D}$ sheets, RSC Adv. 6 (2016) 93384-93393.

[48] I. Stepniak, A. Ciszewski, Grafting effect on the wetting and electrochemical performance of carbon cloth electrode and polypropylene separator in electric double layer capacitor, J. Power Sources 195 (2010) 5130-5137.

[49] J. Foroughi, G.M. Spinks, G.G. Wallace, J. Oh, M.E. Kozlov, S. Fang, T. Mirfakhrai, J.D.W. Madden, M.K. Shin, S.J. Kim, R.H. Baughman, Torsional Carbon Nanotube Artificial Muscles, Science 334 (2011) 494-497. 
[50] A.C.T. van Duin, S. Dasgupta, F. Lorant, W.A. Goddard, ReaxFF: A Reactive Force Field for Hydrocarbons, J. Phys. Chem. A 105 (2001) 9396-9409.

[51] H.M. Aktulga, J.C. Fogarty, S.A. Pandit, A.Y. Grama, Parallel reactive molecular dynamics: Numerical methods and algorithmic techniques, Parallel Comput. 38 (2012) 245259.

[52] S. Plimpton, Fast Parallel Algorithms for Short-Range Molecular Dynamics, J. Comput. Phys. 117 (1995) 1-19. 
Table 1. Atomic \% of the samples: PPy, PPy/rGO and PPy/rGO/ZrO

\begin{tabular}{lcccccc}
\hline Atomic \% & C 1s & O 1s & N 1s & S 2p & Zr 3d & C/N \\
\cline { 1 - 4 } Sample & & & & & & \\
\cline { 1 - 5 } PPy & 70.4 & 15.6 & 12.1 & 1.9 & - & 5.8 \\
PPy/rGO & 66.3 & 21.6 & 7.2 & 4.9 & - & 9.2 \\
PPy/rGO/ZrO 2 & 67.6 & 20.2 & 7.0 & 4.5 & 0.7 & 9.7 \\
\hline
\end{tabular}



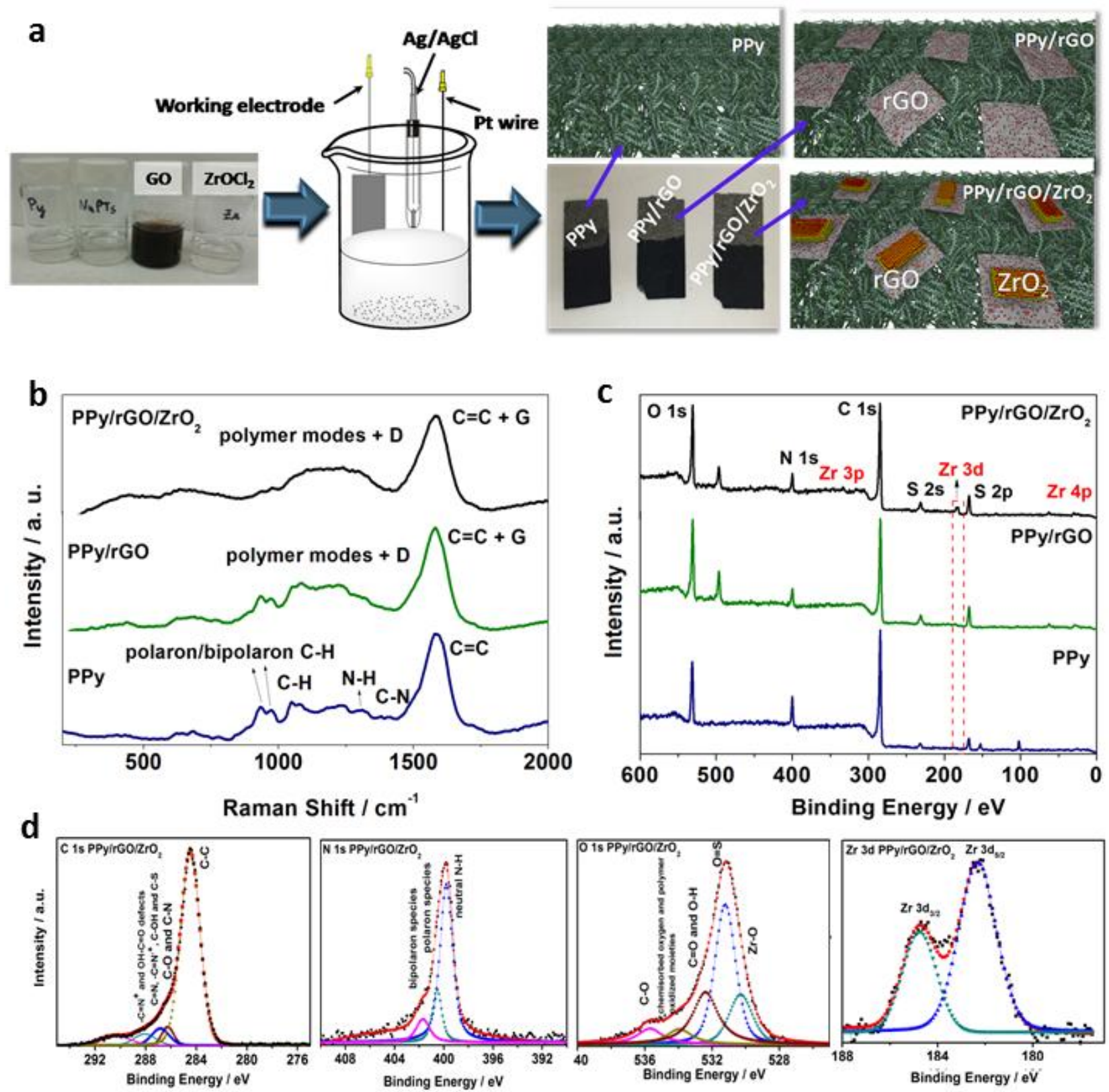

Figure 1. a) Schematic of the synthesis of in-situ 3D electrodes from liquid solution, the electrochemical setup and final electrodes (PPy, PPy/rGO and PPy/rGO/ZrO ${ }_{2}$ ) with schematic of structure, b) Raman spectra for PPy, PPy/rGO and PPy/rGO/ZrO $\mathrm{rO}_{2}$ b) XPS spectra for PPy, $\mathrm{PPy} / \mathrm{rGO}$ and $\mathrm{PPy} / \mathrm{rGO} / \mathrm{ZrO}_{2}$ and d) $\mathrm{PPy} / \mathrm{rGO} / \mathrm{ZrO}_{2}$ high resolution XPS spectra of $\mathrm{C} 1 \mathrm{~s}, \mathrm{~N} 1 \mathrm{~s}$, $\mathrm{O} 1 \mathrm{~s}$ and $\mathrm{Zr} 3 \mathrm{~d}$. Fitted with different binding states. 

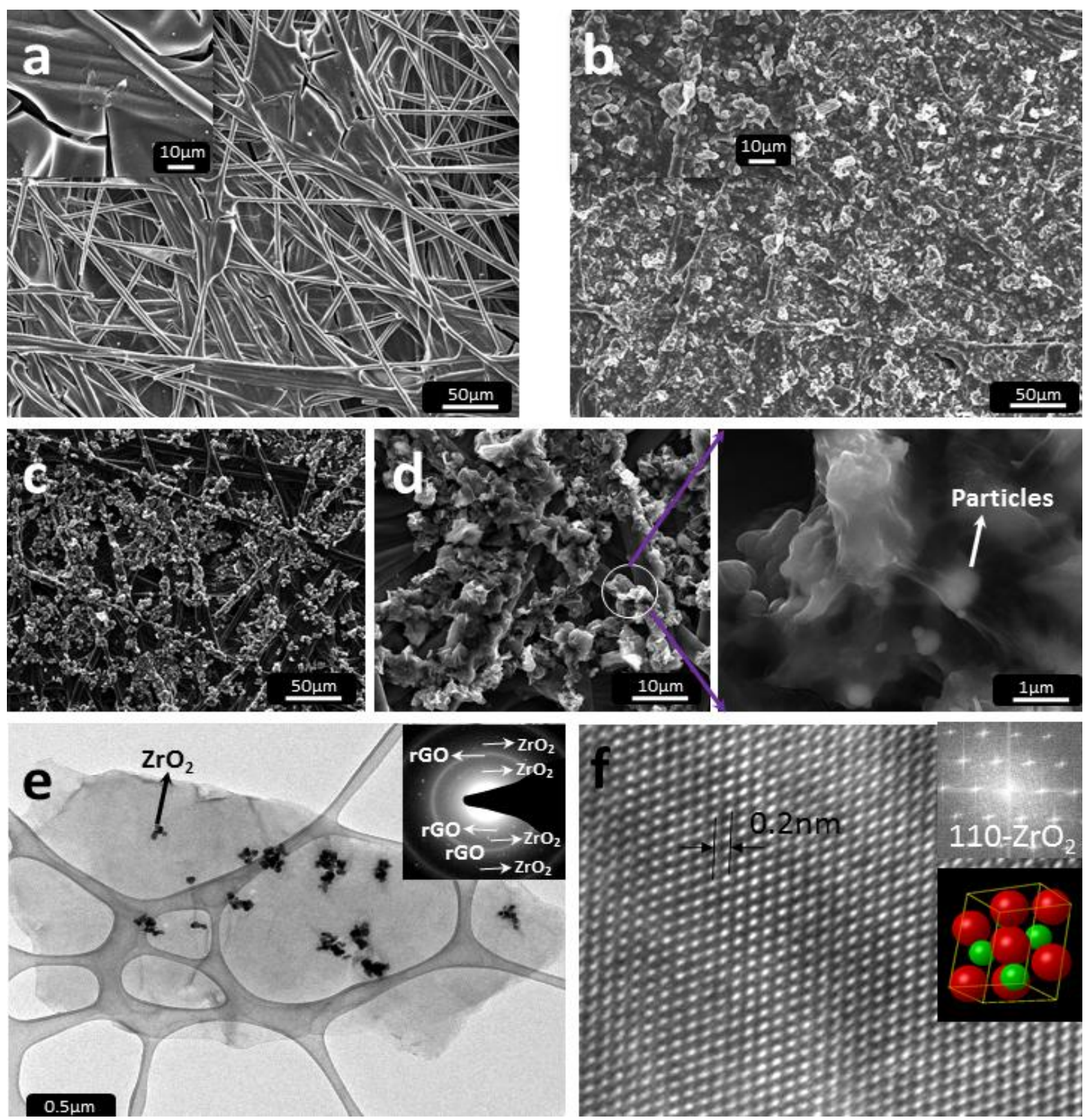

Figure 2. SEM images of the samples: a) pure PPy, with high magnification image of the electrode is shown as inset, b) $\mathrm{PPy} / \mathrm{rGO}$, high magnification image of the electrode is shown as inset $\mathrm{c}-\mathrm{d}$ ) $\mathrm{PPy} / \mathrm{rGO} / \mathrm{ZrO}_{2}$ at different magnifications zooming of different regions, e) bright field TEM images for $\mathrm{rGO} / \mathrm{ZrO}_{2}$ samples with selected area diffraction covering large regions and making refection corresponding to $\mathrm{rGO}$ and $\mathrm{ZrO}_{2}$. d) High Resolution TEM image of the $\mathrm{ZrO}_{2}$ with FFT of the same. 

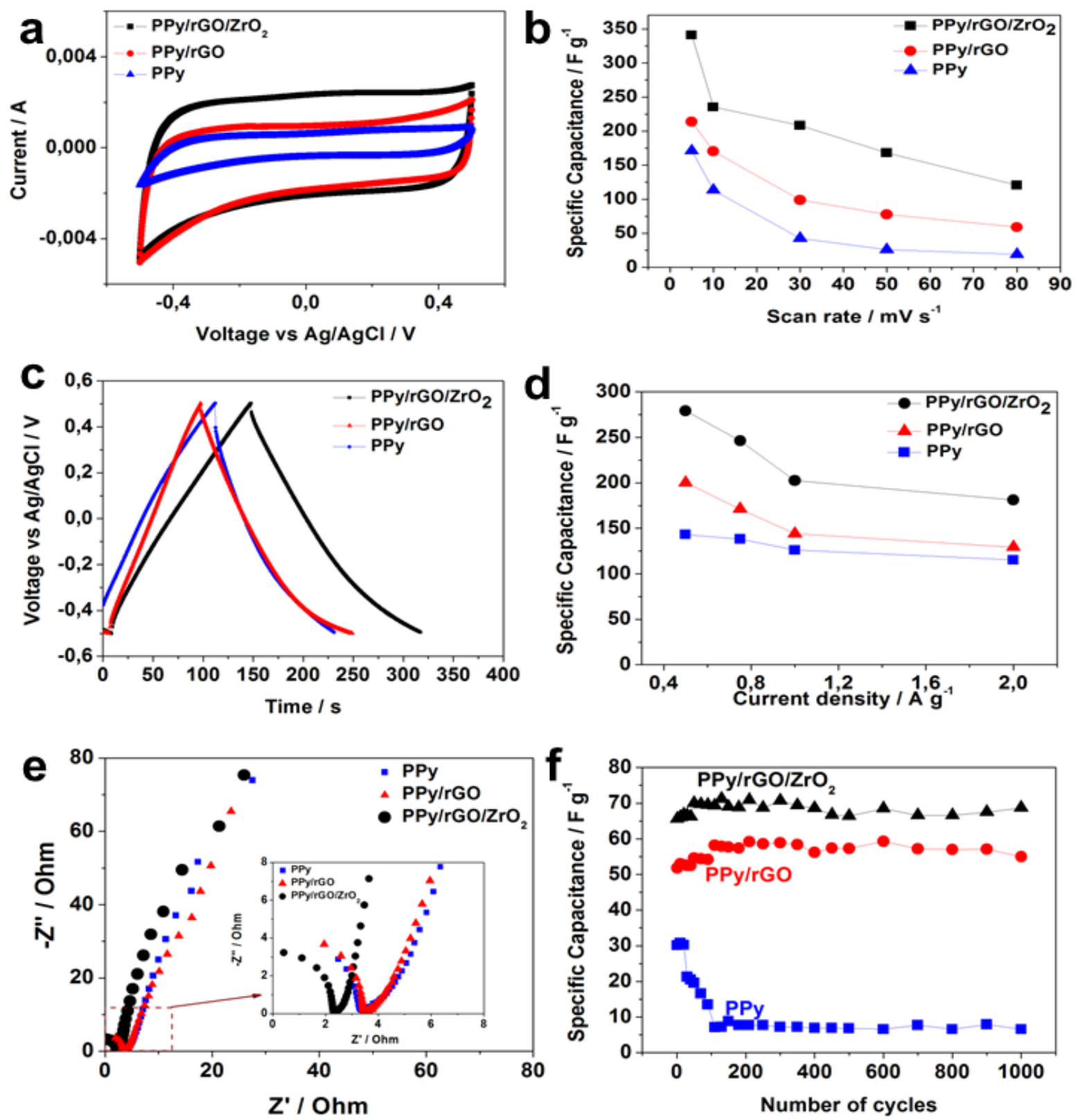

Figure 3. a) $\mathrm{CV}$ curves for PPy, PPy-rGO and PPy-rGO-ZrO $\mathrm{Zr}_{2}$ at $5 \mathrm{mV} \mathrm{s}^{-1}$, b) change in the specific capacitance as a function of scan rates of the samples, c) CD curves for PPy, PPy/rGO and $\mathrm{PPy} / \mathrm{rGO} / \mathrm{ZrO}_{2}$ at $1 \mathrm{~A} \mathrm{~g}^{-1} \mathrm{~d}$ ) change in the specific capacitance as a function of current densities of the samples, e) Nyquist plots for for PPy, PPy/rGO and PPy/rGO/ZrO 2 at high and low frequencies and f) Specific capacitance values as a function of number of cycles. 

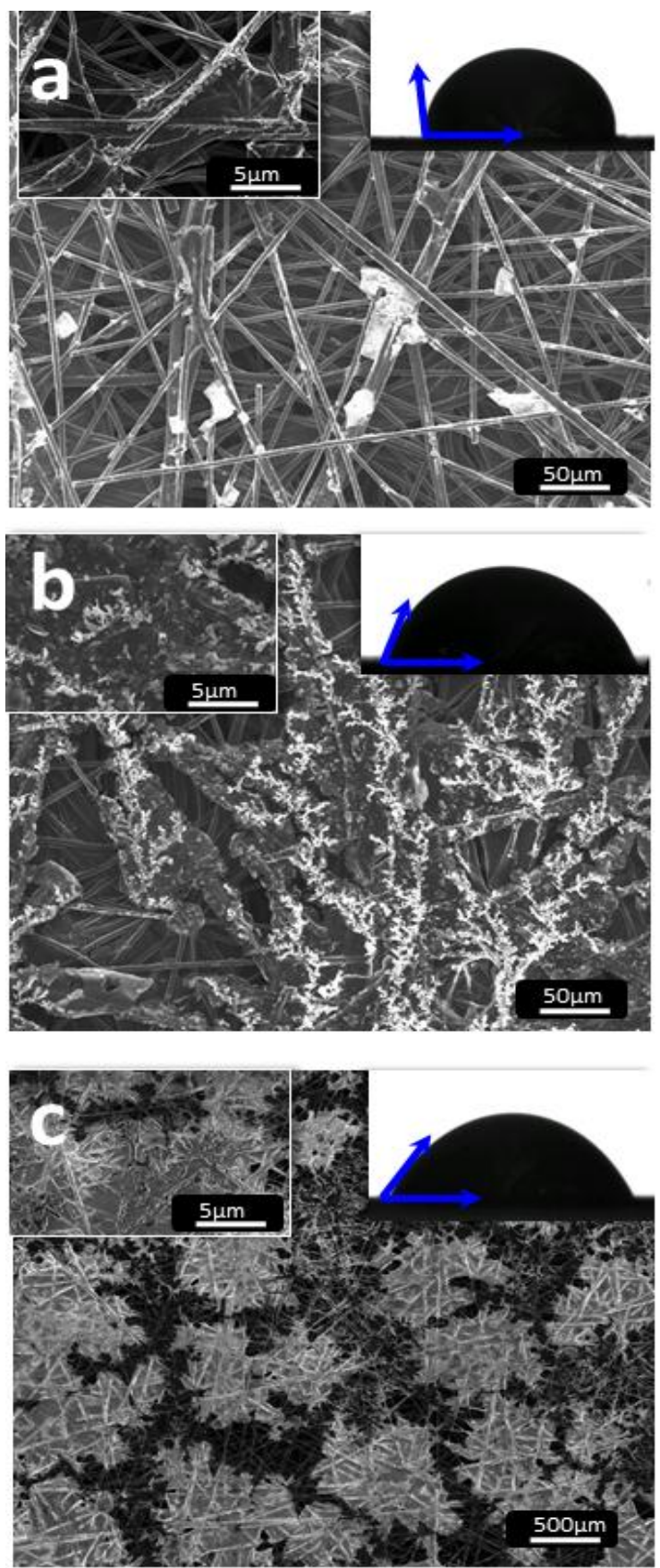

Figure 4. SEM images of the electrode after 1000 cycles test: a) PPy, b) PPy/rGO and c) $\mathrm{PPy} / \mathrm{rGO} / \mathrm{ZrO}_{2}$. The high magnification SEM images of the electrode shown as inset. The Righ inset shows contact angle on electrode and electrolyte. The blue arrow shows the contact angles (PPy, 90, $\mathrm{PPy} / \mathrm{rGO}-70$ and $\mathrm{PPy} / \mathrm{rGO} / \mathrm{ZrO}_{2}-57$ degree). 
a
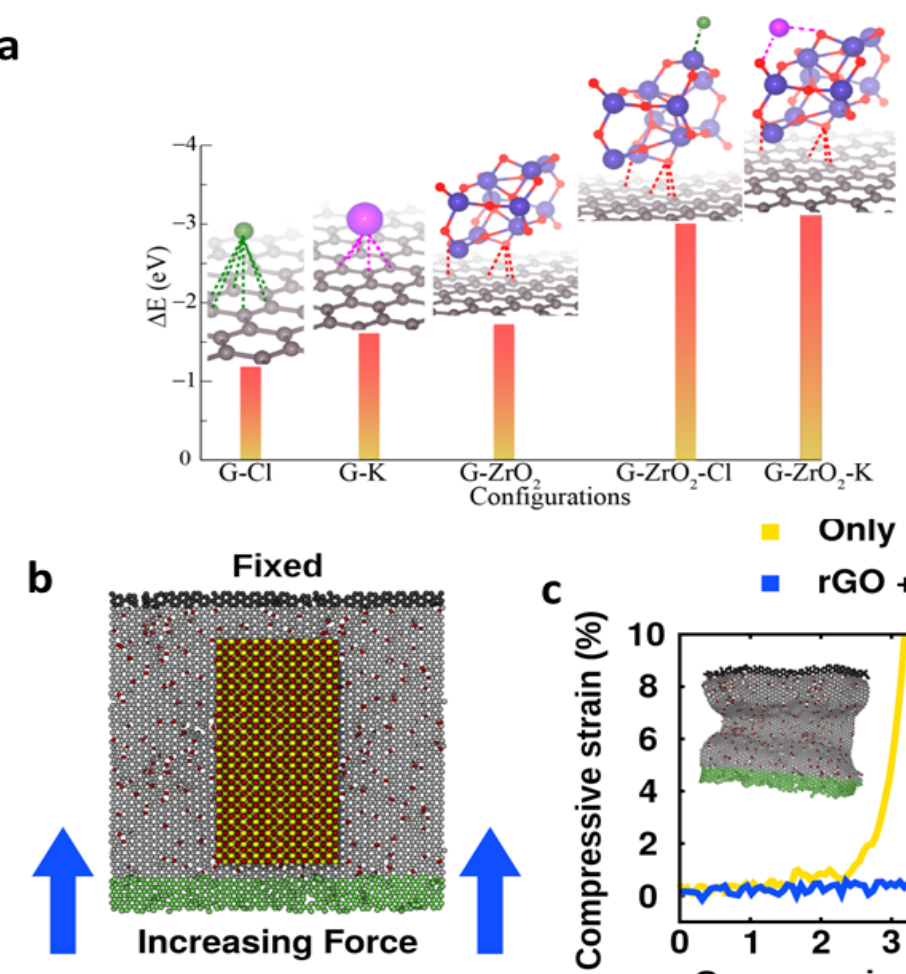

- Only rGo

d
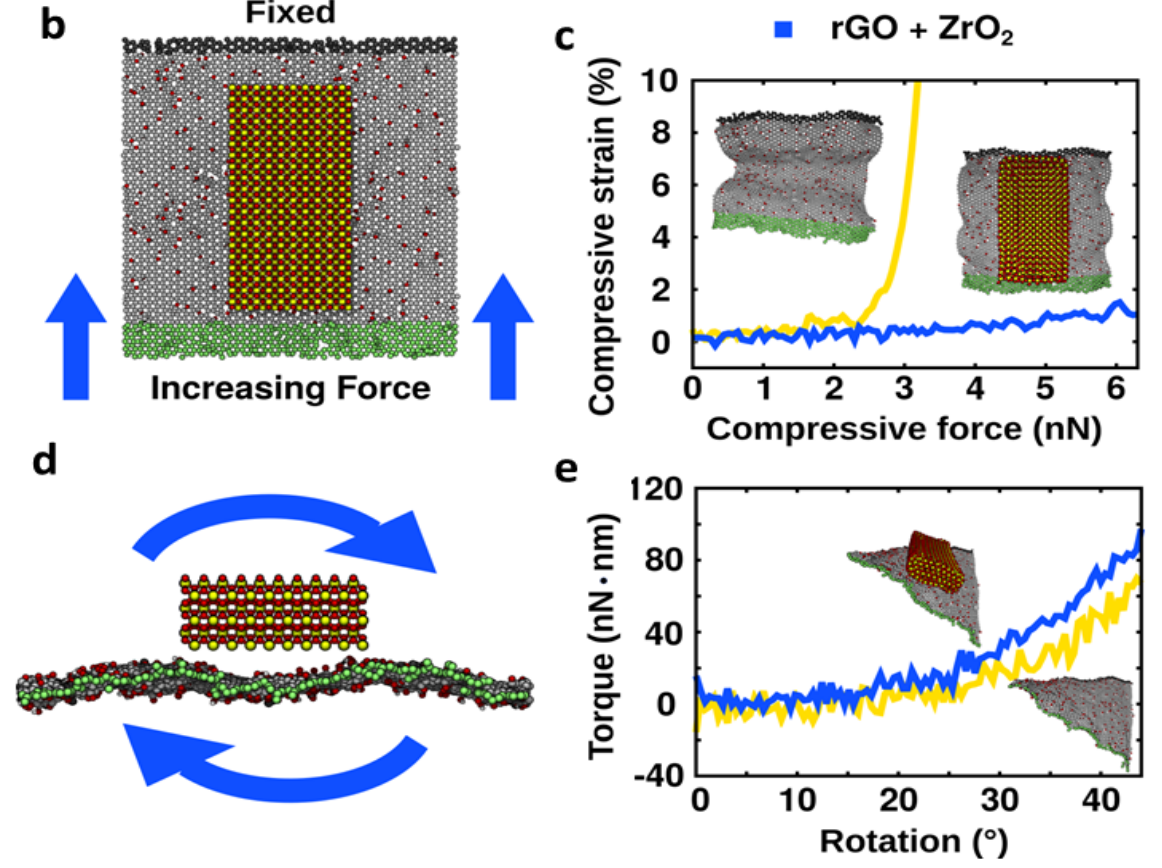

Figure 5.a) DFT calculated interaction energies (Eint) of graphene (G) with $\mathrm{Cl}, \mathrm{K}, \mathrm{ZrO}_{2}$ particle and $\mathrm{G}-\mathrm{ZrO}_{2}$ particle with $\mathrm{Cl}$ and $\mathrm{K}$. The side views of graphene $(\mathrm{G})$ sheet with $\mathrm{Cl}, \mathrm{K}, \mathrm{ZrO}_{2}$ particle and $\mathrm{G}-\mathrm{ZrO}_{2}$ particle with $\mathrm{Cl}$ and $\mathrm{K}$ are shown for clarity (dotted line shows nearest neighbor atoms). $\mathrm{C}, \mathrm{Cl}$ , $\mathrm{K}, \mathrm{Zn}$ and $\mathrm{O}$ atoms are represented in grey, green, magenta, blue and red balls, respectively; b) Setup used in compression tests. The position of atoms in black was fixed, and an increasing force was applied to the ones in green. Other atoms were free to evolve, and we measured the compressive strain. Results are shown in (c), and insets present final configurations after these tests. (d) Setup used in torsion tests. Atoms in green were rotated in the direction indicated by the arrows, while atoms at the opposite side of the sheet were fixed. Other atoms were set free, and we measured the torque required to cause rotation. Results are shown in (e), and insets present final configurations after these tests. 


\section{Table of content}

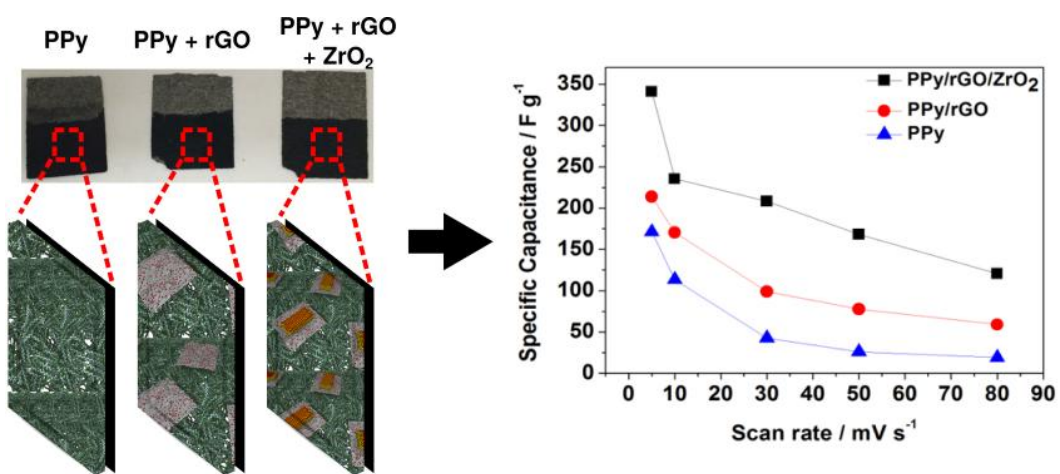

